\title{
Existence of nodal solutions of a nonlinear fourth-order two-point boundary value problem
}

Wenguo Shen ${ }^{1,2}$

\section{Correspondence:}

shenwg1963@126.com

${ }^{1}$ School of Mathematics and

Statistics, Lanzhou University,

Lanzhou 730000, People's Republic

of China

Full list of author information is

available at the end of the article

\begin{abstract}
In this article, we give conditions on parameters $k$, I that the generalized eigenvalue problem $x^{\prime \prime \prime \prime}+k x^{\prime \prime}+\mid x=\lambda h(t) x, 0<t<1, x(0)=x(1)=x^{\prime}(0)=x^{\prime}(1)=0$ possesses an infinite number of simple positive eigenvalues $\left\{\lambda_{k}\right\}_{k=1}^{\infty}$ and to each eigenvalue there corresponds an essential unique eigenfunction $\psi_{k}$ which has exactly $k-1$ simple zeros in $(0,1)$ and is positive near 0 . It follows that we consider the fourth-order twopoint boundary value problem $x^{\prime \prime \prime \prime}+k x^{\prime \prime}+I x=f(t, x), 0<t<1, x(0)=x(1)=x^{\prime}(0)=x^{\prime}$ $(1)=0$, where $f(t, x) \in C([0,1] \times \mathbb{R}, \mathbb{R})$ satisfies $f(t, x) x>0$ for all $x \neq 0, t \in[0,1]$ and $\lim _{|x| \rightarrow 0} f(t, x) / x=a(t), \lim _{|x| \rightarrow+\infty} f(t, x) / x=b(t)$ or $\lim _{x \rightarrow-\infty} f(t, x) / x=0$ and $\lim _{x \rightarrow+\infty} f(t, x) / x$ $=c(t)$ for some $a(t), b(t), c(t) \in C([0,1],(0,+\infty))$ and $t \in[0,1]$. Furthermore, we obtain the existence and multiplicity results of nodal solutions for the above problem. The proofs of our main results are based upon disconjugate operator theory and the global bifurcation techniques.

MSC (2000): 34B15.
\end{abstract}

Keywords: disconjugacy theory, bifurcation, nodal solutions, eigenvalue

\section{Introduction}

The deformations of an elastic beam in equilibrium state with fixed both endpoints can be described by the fourth-order boundary value problem

$$
\begin{aligned}
& x^{\prime \prime \prime \prime}+l x=\lambda h(t) f(x), 0<t<1, \\
& x(0)=x(1)=x^{\prime}(0)=x^{\prime}(1)=0,
\end{aligned}
$$

where $f: \mathbb{R} \rightarrow \mathbb{R}$ is continuous, $\lambda \in \mathbb{R}$ is a parameter and $l$ is a given constant. Since the problem (1.1) cannot transform into a system of second-order equation, the treatment method of second-order system does not apply to the problem (1.1). Thus, existing literature on the problem (1.1) is limited. Recently, when $l=0$, the existence and multiplicity of positive solutions of the problem (1.1) has been studied by several authors, see Agarwal and Chow [1], Ma and Wu [2], Yao [3,4] and Korman [5]. Especially, when $l \neq 0, l$ satisfying $(H 1)$ and $h(t)$ satisfying $(H 2), \mathrm{Xu}$ and Han [6] studied the existence of nodal solutions of the problem (1.1) by applying bifurcation techniques, where

(H1) $l \in\left(-\pi^{4}, \pi^{4} / 64\right)$ is given constant.

$(H 2) h \in C([0,1],[0, \infty))$ with $h(t) \equiv 0$ on any subinterval of $[0,1]$.

(c) 2012 Shen; licensee Springer. This is an open access article distributed under the terms of the Creative Commons Attribution License (http://creativecommons.org/licenses/by/2.0), which permits unrestricted use, distribution, and reproduction in any medium, provided the original work is properly cited. 
Motivated by [6], we consider the existence of nodal solutions of general fourthorder boundary value problem

$$
\begin{aligned}
& x^{\prime \prime \prime \prime}+k x^{\prime \prime}+l x=f(t, x), 0<t<1, \\
& x(0)=x(1)=x^{\prime}(0)=x^{\prime}(1)=0,
\end{aligned}
$$

and under the assumptions:

(A1) One of following conditions holds

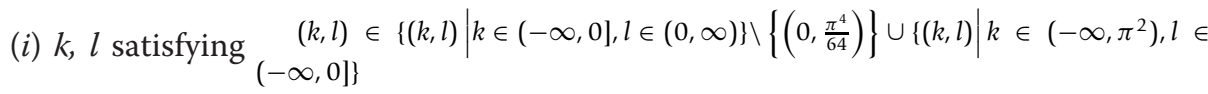
are given constants with

$$
\pi^{2}\left(k-\pi^{2}\right)<l \leq \frac{1}{4}\left(k-\frac{\pi^{2}}{4}\right)^{2}
$$

(ii) $k, l$ satisfying $(k, l) \in\left\{(k, l) \mid k \in\left(0, \frac{\pi^{2}}{2}\right), l \in(0, \infty)\right\}$ are given constants with

$$
\frac{1}{4}\left(\pi^{2} k-\frac{\pi^{4}}{4}\right)<l \leq \frac{1}{4} k^{2} .
$$

(A2) $f(t, x) \in C([0,1] \times \mathbb{R}, \mathbb{R})$ satisfies $f(t, x) x>0$ for all $x \neq 0$ and $t \in[0,1]$.

(A3) There exists $a(t) \in C([0,1],(0, \infty))$ such that

$$
\lim _{|x| \rightarrow 0} \frac{f(t, x)}{x}=a(t), \forall t \in[0,1] .
$$

(A4) There exists $b(t) \in C([0,1],(0, \infty))$ such that

$$
\lim _{|x| \rightarrow \infty} \frac{f(t, x)}{x}=b(t), \forall t \in[0,1] .
$$

(A5) There exists $c(t) \in C([0,1],(0, \infty))$ such that

$$
\lim _{x \rightarrow-\infty} \frac{f(t, x)}{x}=0, \lim _{x \rightarrow+\infty} \frac{f(t, x)}{x}=c(t), \forall t \in[0,1] .
$$

However, in order to use bifurcation technique to study the nodal solutions of the problem (1.2), we first prove that the generalized eigenvalue problem

$$
\begin{aligned}
& x^{\prime \prime \prime \prime}+k x^{\prime \prime}+l x=\lambda h(t) x, 0<t<1, \\
& x(0)=x(1)=x^{\prime}(0)=x^{\prime}(1)=0,
\end{aligned}
$$


(where $h$ satisfies $(H 2)$ ) has an infinite number of positive eigenvalues

$$
0<\lambda_{1}(h)<\lambda_{2}(h)<\cdots<\lambda_{k}(h)<\lambda_{k+1}(h)<\cdots
$$

and each eigenvalue corresponding an essential unique eigenfunction $\psi_{k}$ which has exactly $k$ - 1 simple zeros in $(0,1)$ and is positive near 0. Fortunately, Elias [7] developed a theory on the eigenvalue problem

$$
\begin{gathered}
\mathcal{L} y+\lambda h(t) y=0, \\
\left(\mathcal{L}_{i} y\right)(a)=0, i \in\left\{i_{1}, \ldots, i_{k}\right\}, \\
\left(\mathcal{L}_{j} y\right)(b)=0, j \in\left\{j_{1}, \ldots, j_{n-k}\right\},
\end{gathered}
$$

where

$$
\begin{gathered}
\mathcal{L}_{0} \gamma=\rho_{0} \gamma, \\
\mathcal{L}_{i} y=\rho_{i}\left(\mathcal{L}_{i-1} \gamma\right)^{\prime}, i=1, \ldots, n, \\
\mathcal{L} \gamma=\mathcal{L}_{n} \gamma,
\end{gathered}
$$

and $\rho_{i} \in C^{n-i}[a, b]$ with $\rho_{i}>0(i=0,1, \ldots, n)$ on $[a, b] . \mathcal{L}_{0} y, \ldots ., \mathcal{L}_{n-1} y$ are called the quasi-derivatives of $y(t)$. To apply Elias's theory, we have to prove that (1.8) can be rewritten to the form of (1.10), that is, the linear operator

$$
L[x]:=x^{\prime \prime \prime \prime}+k x^{\prime \prime}+l x
$$

has a factorization of the form

$$
L[x]=l_{4}\left(l_{3}\left(l_{2}\left(l_{1}\left(l_{0} x\right)^{\prime}\right)^{\prime}\right)^{\prime}\right)^{\prime}
$$

on $[0,1]$, where $l_{i} \in C^{4-i}[0,1]$ with $l_{i}>0(i=0,1,2,3,4)$ on $[0,1]$, and $x(0)=x(1)=x$ ${ }^{\prime}(0)=x^{\prime}(1)=0$ if and only if

$$
\left(l_{0} x\right)(0)=\left(l_{0} x\right)(1)=\left(l_{1} x\right)(0)=\left(l_{1} x\right)(1)=0 .
$$

This can be achieved under (A1) by using the disconjugacy theory in [8].

The rest of the article is arranged as follows: In Section 2, we state some disconjugacy theory which can be used in this article, and then show that $(A 1)$ implies the equation

$$
L[x]=0
$$

is disconjugate on $[0,1]$, and establish some preliminary properties on the eigenvalues and eigenfunctions of the generalized eigenvalue problem (1.8). Finally in Section 3 , we state and prove our main results (Theorems 3.1 and 3.2 ).

Remark 1.1. If we let $k=0$, then the condition $(A 1)$ reduces to $(H 1)$ in [6].

Remark 1.2. Since the function $f(t, x)$ is more general than the function $h(t) f(x)$ in [6], then the problem considered in this article is more general than the problem in [6].

Remark 1.3. If we let $k=0$ and $f(t, x)=\lambda h(t) f(x)$, then Theorem 3.2 reduces to [[6], Theorem 3.1].

Remark 14. For other results on the existence and multiplicity of positive solutions and nodal solutions for the boundary value problems of fourth-order ordinary differential equations based on bifurcation techniques, see [9-14]s and their references. 


\section{Preliminary results}

Let

$$
\mathbf{L}[\gamma]=\gamma^{(n)}+p_{1}(t) y^{(n-1)}+\cdots+p_{n}(t) y=0
$$

be $n$ th-order linear differential equation whose coefficients $p_{k}(\cdot)(k=1, \ldots, n)$ are continuous on an interval $I$.

Definition 2.1 [[8], Definition 0.2, p. 2]. Equation (2.1) is said to be disconjugate on an interval $I$ if no nontrivial solution has $n$ zeros on $I$, multiple zeros being counted according to their multiplicity.

Lemma 2.2 [[8], Theorem 0.7, p. 3]. Equation (2.1) is disconjugate on a compact interval I if and only if there exists a basis of solutions $y_{0}, \ldots, y_{n-1}$ such that

$$
w_{k}:=w_{k}\left(y_{0}, \ldots, \gamma_{k-1}\right)=\left|\begin{array}{ccc}
y_{0} & \cdots & y_{k-1} \\
\vdots & & \vdots \\
y_{0}^{(k-1)} & \cdots & y_{k-1}^{(k-1)}
\end{array}\right|>0(k=1, \ldots, n)
$$

on I. A disconjugate operator $\boldsymbol{L}[y]=y^{(n)}+p_{1}(t) y^{(n-1)}+\ldots+p_{n}(t) y$ can be written as

$$
L[\gamma]=\rho_{n} D\left(\rho_{n-1}\left(\cdots D\left(\rho_{1} D\left(\rho_{0} \gamma\right)\right) \cdots\right)\right), D=\frac{d}{d t},
$$

where $\rho_{k} \in C^{n-k}(I)(k=0,1, \ldots, n)$ and

$$
\rho_{0}=\frac{1}{w_{1}}, \rho_{1}=\frac{w_{1}^{2}}{w_{2}}, \rho_{k}=\frac{w_{k}^{2}}{w_{k-1} w_{k+1}}, k=2, \ldots, n-1,
$$

and $\rho_{0} \rho_{1} \ldots \rho_{n} \equiv 1$.

Lemma 2.3 [[8], Theorem 0.13, p. 9]. Green's function $G(t, s)$ of the disconjugate equation (2.3) and the two-point boundary value conditions

$$
\begin{gathered}
y^{(i)}(a)=0, i=0, \ldots, k-1, \\
y^{(j)}(b)=0, j=0, \ldots, n-k-1
\end{gathered}
$$

satisfies

$$
(-1)^{n-k} G(t, s)>0, \forall(t, s) \in(a, b) \times(a, b) .
$$

Now using Lemmas 2.2 and 2.3, we will prove some preliminary results.

Theorem 2.4. Let (A1) hold. Then

(i) $L[x]=0$ is disconjugate on $[0,1]$, and $L[x]$ has a factorization

$$
L[x]=\rho_{4}\left(\rho_{3}\left(\rho_{2}\left(\rho_{1}\left(\rho_{0} x\right)^{\prime}\right)^{\prime}\right)^{\prime}\right)^{\prime}
$$

where $\rho_{k} \in C^{4-k}[0,1]$ with $\rho_{k}>0(k=0,1,2,3,4)$.

(ii) $x(0)=x(1)=x^{\prime}(0)=x^{\prime}(1)=0$ if and only if

$$
\left(L_{0} x\right)(0)=\left(L_{1} x\right)(0)=\left(L_{0} x\right)(1)=\left(L_{1} x\right)(1)=0,
$$


where

$$
\begin{gathered}
L_{0} x=\rho_{0} x \\
L_{i} x=\rho_{i}\left(L_{i-1} x\right)^{\prime}, i=1,2,3,4 .
\end{gathered}
$$

Proof of Theorem 2.4. We divide the proof into nine cases.

Case 1. $(k, l) \in\left\{(k, l) \mid k \in(-\infty, 0], l \in\left(\frac{1}{4} k^{2}, \frac{1}{4}\left(k-\frac{\pi^{2}}{4}\right)^{2}\right)\right\} \backslash\left\{\left(0, \frac{\pi^{4}}{64}\right)\right\}$.

In the case, we have corresponding $L[x]=x^{\prime \prime \prime \prime}+k x^{\prime \prime}+l x=0$ that the equation $\lambda^{4}+$ $k \lambda^{2}+l x=0$ has 4 roots $\lambda_{1}=m_{1}+m_{2} i, \lambda_{2}=m_{1}-m_{2} i, \lambda_{3}=-m_{1}+m_{2} i$, and $\lambda_{4}=-m_{1}-$ $m_{2} i$, where

$$
m_{1}=\frac{\sqrt{-k+\sqrt{4 l}}}{2}, m_{2}=\frac{\sqrt{k+\sqrt{4 l}}}{2}, m_{2} \leq m_{1}
$$

Combining $\frac{1}{4} k^{2}<l \leq \frac{1}{4}\left(k-\frac{\pi^{2}}{4}\right)^{2}$ with (2.10), we have $0<m_{2} \leq \frac{\pi}{4}$. Thus, we get that either the following (1) or (2) holds:

(1) $0 \leq m_{2} t<\frac{\pi}{4} \Rightarrow \tan m_{2} t<1 \leq \frac{m_{1}}{m_{2}}$, for $m_{2} \in\left(0, \frac{\pi}{4}\right), t \in[0,1]$;

(2) $0 \leq m_{2} t \leq \frac{\pi}{4} \Rightarrow \tan m_{2} t \leq 1<\frac{m_{1}}{m_{2}}$, for $m_{2}=\frac{\pi}{4}, t \in[0,1]$.

Furthermore, it is easy to check that

$$
\cos m_{2} t>0, m_{1} \cos m_{2} t-m_{2} \sin m_{2} t>0, \forall t \in[0,1] .
$$

Take

$$
\begin{gathered}
x_{0}(t)=e^{-m_{1} t} \cos m_{2} t, x_{1}(t)=e^{-m_{1} t} \sin m_{2} t \\
x_{2}(t)=e^{m_{1} t} \cos m_{2} t, x_{3}(t)=e^{m_{1} t} \sin m_{2} t .
\end{gathered}
$$

It is easy to check that $x_{0}(t), x_{1}(t), x_{2}(t)$, and $x_{3}(t)$ form a basis of solutions of $L[x]=$ 0 . By simple computation, we have

$$
\begin{gathered}
w_{1}=\frac{\cos m_{2} t}{e^{m_{1} t}}, w_{2}=\frac{m_{2}}{e^{2 m_{1} t}}, \\
w_{3}=\frac{4 m_{1} m_{2}\left(m_{1} \cos m_{2} t-m_{2} \sin m_{2} t\right)}{e^{m_{1} t}}, w_{4}=16 m_{1}^{2} m_{2}^{2}\left(m_{1}^{2}+m_{2}^{2}\right) .
\end{gathered}
$$

This together with (2.11) implies that $w_{i}>0(i=1,2,3,4)$ on $[0,1]$.

By Lemma 2.2, $L[x]=0$ is disconjugate on $[0,1]$, and $L[x]$ has a factorization

$$
\begin{aligned}
& x^{\prime \prime \prime \prime}+k x^{\prime \prime}-l x=\frac{4 m_{1} m_{2}\left(m_{1}^{2}+m_{2}^{2}\right) e^{m_{1} t}}{m_{1} \cos m_{2} t-m_{2} \sin m_{2} t}\left(\frac{\left(m_{1} \cos m_{2} t-m_{2} \sin m_{2} t\right)^{2}}{m_{2}\left(m_{1}^{2}+m_{2}^{2}\right)}\right. \\
& \left.\times\left(\frac{m_{2}}{4 m_{1} \cos m_{2} t\left(m_{1} \cos m_{2} t-m_{2} \sin m_{2} t\right) e^{2 m_{1} t}}\left(\frac{\cos ^{2} m_{1} t}{m_{2}}\left(\frac{e^{m_{1} t}}{\cos m_{2} t} x\right)^{\prime}\right)^{\prime}\right)^{\prime}\right)^{\prime}
\end{aligned}
$$


and accordingly

$$
\begin{gathered}
L_{0} x=\rho_{0} x=\frac{e^{m_{1} t}}{\cos m_{2} t} x, \\
L_{1} x=\frac{\cos ^{2} m_{2} t}{m_{2}}\left(\frac{e^{m_{1} t}}{\cos m_{2} t} x\right)^{\prime}=\frac{e^{m_{1} t}}{m_{2}}\left(m_{1} \cos m_{2} t+m_{2} \sin m_{2} t\right) x+\frac{e^{m_{1} t} \cos m_{2} t}{m_{2}} x^{\prime} .
\end{gathered}
$$

Using (2.15), we conclude that $x(0)=x(1)=x^{\prime}(0)=x^{\prime}(1)=0$ is equivalent to (2.8).

Case 2. $k \in(-\infty, 0)$ and $l=\frac{1}{4} k^{2}$.

In the case, applying the similar method used in Case 1, we take

$$
x_{0}(t)=e^{-m t}, x_{1}(t)=t e^{-m t}, x_{2}(t)=e^{m t}, x_{3}(t)=t e^{m t},
$$

where $m=\sqrt{-\frac{k}{2}}$.

It is easy to check that $x_{0}(t), x_{1}(t), x_{2}(t)$, and $x_{3}(t)$ form a basis of solutions of $L[x]=$ 0 .

By simple computation, we have

$$
w_{1}=e^{-m t}, w_{2}=e^{-2 m t}, w_{3}=4 m^{2} e^{-m t}, w_{4}=16 m^{4} .
$$

Clearly, $w_{i}>0(i=1,2,3,4)$ on $[0,1]$.

By Lemma 2.2, $L[x]=0$ is disconjugate on $[0,1]$, and $L[x]$ has a factorization

$$
x^{\prime \prime \prime \prime}+k x^{\prime \prime}-l x=\frac{4 m^{2} e^{m t}}{1}\left(\frac{1}{1}\left(\frac{1}{4 m^{2} e^{2 m t}}\left(\frac{1}{1}\left(\frac{e^{m t}}{1} x\right)^{\prime}\right)^{\prime}\right)^{\prime}\right)^{\prime}
$$

and accordingly

$$
L_{0} x=\rho_{0} x=e^{m t} x, L_{1} x=\rho_{1}\left(L_{0} x\right)^{\prime}=e^{m t}\left(m x+x^{\prime}\right) .
$$

Using (2.19), we conclude that $x(0)=x(1)=x^{\prime}(0)=x^{\prime}(1)=0$ is equivalent to $(2.8)$.

Case $3 . k \in(-\infty, 0)$ and $0<l<\frac{1}{4} k^{2}$.

In the case, we take

$$
x_{0}(t)=e^{-m_{2} t}, x_{1}(t)=e^{m_{2} t}, x_{2}(t)=e^{-m_{1} t}, x_{3}(t)=e^{m_{1} t},
$$

where $m_{1}=\sqrt{\frac{-k+\sqrt{k^{2}-4 l}}{2}}>0, m_{2}=\sqrt{\frac{-k-\sqrt{k^{2}-4 l}}{2}}>0, m_{1}>m_{2}$,

It is easy to check that $x_{0}(t), x_{1}(t), x_{2}(t)$, and $x_{3}(t)$ form a basis of solutions of $L[x]=$ 0 .

By simple computation, we have

$$
\begin{gathered}
w_{1}=e^{-m_{2} t}, w_{2}=2 m_{2}, \\
w_{3}=2 m_{2}\left(m_{1}^{2}-m_{2}^{2}\right) e^{-m_{1} t}, w_{4}=4 m_{1} m_{2}\left(m_{1}^{2}-m_{2}^{2}\right)^{2} .
\end{gathered}
$$

Clearly, $w_{i}>0(i=1,2,3,4)$ on $[0,1]$. 
By Lemma 2.2, $L[x]=0$ is disconjugate on $[0,1]$, and $L[x]$ has a factorization

$$
\begin{aligned}
& x^{\prime \prime \prime \prime}+k x^{\prime \prime}+l x \\
& =2 m_{1}\left(m_{1}^{2}-m_{2}^{2}\right) e^{m_{1} t}\left(\frac{1}{2 m_{1} e^{2 m_{1} t}}\left(\frac{2 m_{2} e^{\left(m_{1}+m_{2}\right) t}}{m_{1}^{2}-m_{2}^{2}}\left(\frac{1}{2 m_{2} e^{2 m_{2} t}}\left(e^{m_{2} t} x\right)^{\prime}\right)^{\prime}\right)^{\prime}\right)^{\prime}
\end{aligned}
$$

and accordingly

$$
L_{0} x=\rho_{0} x=e^{m_{2} t} x, L_{1} x=\rho_{1}\left(L_{0} x\right)=\frac{1}{2 m_{2} e^{m_{2} t}}\left(m_{2} x+x^{\prime}\right) .
$$

Using (2.23), we conclude that $x(0)=x(1)=x^{\prime}(0)=x^{\prime}(1)=0$ is equivalent to (2.8).

Case 4. $k \in(-\infty, 0), l=0$.

In the case, we take

$$
x_{0}(t)=1, x_{1}(t)=1+t, x_{2}(t)=e^{-m t}, x_{3}(t)=e^{m t},
$$

where $m=\sqrt{-k}>0$.

It is easy to check that $x_{0}(t), x_{1}(t), x_{2}(t)$, and $x_{3}(t)$ form a basis of solutions of $L[x]=$ 0 .

By simple computation, we have

$$
w_{1}=1, w_{2}=1, w_{3}=m^{2} e^{-m t}, w_{4}=2 m^{5} .
$$

Clearly, $w_{i}>0(i=1,2,3,4)$ on $[0,1]$.

By Lemma 2.2, $L[x]=0$ is disconjugate on $[0,1]$, and $L[x]$ has a factorization

$$
x^{\prime \prime \prime \prime}+k x^{\prime \prime}+l x=2 m^{3} e^{m t}\left(\frac{1}{2 m e^{2 m t}}\left(\frac{e^{m t}}{m^{2}}\left(\frac{1}{1}\left(\frac{1}{1} x\right)^{\prime}\right)^{\prime}\right)^{\prime}\right)^{\prime}
$$

and accordingly

$$
L_{0} x=\rho_{0} x=x, L_{1} x=x^{\prime} .
$$

Using (2.27), we conclude that $x(0)=x(1)=x^{\prime}(0)=x^{\prime}(1)=0$ is equivalent to (2.8).

Case $5 . k=0, l=0$. The case is obvious.

Case 6. $k \in\left(0, \pi^{2}\right), l=0$.

In the case, we take

$$
x_{0}(t)=1, x_{1}(t)=1+t, x_{2}(t)=-\sin m(t+\sigma), x_{3}(t)=\cos m(t+\sigma),
$$

where $m=\sqrt{k}>0, \sigma$ is a positive constant. Clearly, $m \in(0, \pi)$ and then

$$
\sin m(t+\sigma)>0, \forall t \in[0,1] \text {. }
$$

It is easy to check that $x_{0}(t), x_{1}(t), x_{2}(t)$, and $x_{3}(t)$ form a basis of solutions of $L[x]=$ 0 .

By simple computation, we have

$$
w_{1}=1, w_{2}=1, w_{3}=m_{2} \sin m(t+\sigma), w_{4}=m^{5} .
$$

Clearly, $w_{i}>0(i=1,2,3,4)$ on $[0,1]$. 
By Lemma 2.2, $L[x]=0$ is disconjugate on $[0,1]$, and $L[x]$ has a factorization

$$
x^{\prime \prime \prime \prime}+k x^{\prime \prime}+l x=\frac{m^{3}}{\sin m(t+\sigma)}\left(\frac{\sin ^{2} m(t+\sigma)}{m}\left(\frac{1}{m^{2} \sin m(t+\sigma)}\left(\frac{1}{1}\left(\frac{1}{1} x\right)\right)^{\prime}\right)^{\prime}\right)^{\prime}
$$

and accordingly

$$
L_{0} x=\rho_{0} x=x, L_{1} x=x^{\prime} .
$$

Using (2.32), we conclude that $x(0)=x(1)=x^{\prime}(0)=x^{\prime}(1)=0$ is equivalent to (2.8). Case 7. $\pi^{2}\left(k-\pi^{2}\right)<l<0$.

In the case, we take

$$
x_{0}(t)=e^{-m_{1} t}, x_{1}(t)=e^{m_{1} t}, x_{2}(t)=-\sin m_{2}(t+\sigma), x_{3}(t)=\cos m_{2}(t+\sigma),
$$

where $m_{1}=\sqrt{\frac{-k+\sqrt{k^{2}-4 l}}{2}>0}, m_{2}=\sqrt{\frac{k+\sqrt{k^{2}-4 l}}{2}>0}, \sigma$ is a positive constant. Clearly, $m_{2} \in(0, \pi)$ and then

$$
\sin m_{2}(t+\sigma)>0, \forall t \in[0,1] .
$$

It is easy to check that $x_{0}(t), x_{1}(t), x_{2}(t)$, and $x_{3}(t)$ form a basis of solutions of $L[x]=$ 0 . By simple computation, we have

$$
\begin{gathered}
w_{1}=e^{-m_{1} t}, w_{2}=2 m_{1}, \\
w_{3}=2 m_{1}\left(m_{1}^{2}+m_{2}^{2}\right) \sin m_{2}(t+\sigma), w_{4}=2 m_{1} m_{2}\left(m_{1}^{2}+m_{2}^{2}\right)^{2} .
\end{gathered}
$$

Clearly, $w_{i}>0(i=1,2,3,4)$ on $[0,1]$.

By Lemma 2.2, $L[x]=0$ is disconjugate on $[0,1]$, and $L[x]$ has a factorization

$$
\begin{aligned}
& x^{\prime \prime \prime \prime}+k x^{\prime \prime}+l x \\
& =\frac{m_{2}\left(m_{1}^{2}+m_{2}^{2}\right)}{\sin m_{2}(t+\sigma)}\left(\frac{\sin ^{2} m_{2}(t+\sigma)}{m_{2}}\left(\frac{2 m_{1} e^{m_{1} t}}{\left(m_{1}^{2}+m_{2}^{2}\right) \sin m_{2}(t+\sigma)}\left(\frac{1}{2 m_{1} e^{2 m_{1} t}}\left(e^{m_{1} t} x\right)^{\prime}\right)^{\prime}\right)^{\prime}\right)^{\prime}
\end{aligned}
$$

and accordingly

$$
L_{0} x=\rho_{0} x=e^{m_{1} t} x, L_{1} x=\rho_{1}\left(L_{0} x\right)=\frac{1}{2 m_{1} e^{m_{1} t}}\left(m_{1} x+x^{\prime}\right) .
$$

Using (2.37), we conclude that $x(0)=x(1)=x^{\prime}(0)=x^{\prime}(1)=0$ is equivalent to (2.8).

Case $8 . k \in\left(0, \frac{\pi^{2}}{2}\right)$ and $l=\frac{1}{4} k^{2}$

In the case, we take

$$
\begin{gathered}
x_{0}(t)=\cos m(t+\sigma), x_{1}(t)=\sin m(t+\sigma), \\
x_{2}(t)=-t \cos m(t+\sigma), x_{3}(t)=-t \sin m(t+\sigma),
\end{gathered}
$$

where $m=\sqrt{\frac{k}{2}}, \sigma$ is a positive constant. Clearly, $m \in\left(0, \frac{\pi}{2}\right)$ and then

$$
\cos m(t+\sigma)>0, \sin m(t+\sigma)>0, \forall t \in[0,1]
$$

It is easy to check that $x_{0}(t), x_{1}(t), x_{2}(t)$, and $x_{3}(t)$ form a basis of solutions of $L[x]=$ 0 . By simple computation, we have 


$$
w_{1}=\cos m(t+\sigma), w_{2}=m, w_{3}=2 m^{2} \sin m(t+\sigma), w_{4}=4 m^{4} .
$$

Clearly, $w_{i}>0(i=1,2,3,4)$ on $[0,1]$.

By Lemma $2.2, L[x]=0$ is disconjugate on $[0,1]$, and $L[x]$ has a factorization

$$
\begin{aligned}
& x^{\prime \prime \prime \prime}+k x^{\prime \prime}+l x \\
& =\frac{2 m^{2}}{\sin m(t+\sigma)}\left(\frac{\sin ^{2} m(t+\sigma)}{m}\left(\frac{1}{\sin 2 m(t+\sigma)}\left(\frac{\cos ^{2} m(t+\sigma)}{m}\left(\frac{1}{\cos m(t+\sigma)} x\right)\right)^{\prime}\right)^{\prime}\right)^{\prime}
\end{aligned}
$$

and accordingly

$$
\begin{gathered}
L_{0} x=\rho_{0} x=\frac{1}{\cos m(t+\sigma)} x, \\
L_{1} x=\rho_{1}\left(L_{0} x\right)^{\prime}=\frac{1}{m}\left(m \sin m(t+\sigma) \cdot x+\cos m(t+\sigma) \cdot x^{\prime}\right) .
\end{gathered}
$$

Using (2.42), we conclude that $x(0)=x(1)=x^{\prime}(0)=x^{\prime}(1)=0$ is equivalent to $(2.8)$.

Case 9. $k \in\left(0, \frac{\pi^{2}}{2}\right), l \in(0, \infty), \frac{1}{4}\left(\pi^{2} k-\frac{\pi^{4}}{4}\right)<l<\frac{1}{4} k^{2}$

In the case, we take

$$
x_{0}(t)=\cos m_{1} t, x_{1}(t)=\sin m_{1} t, x_{2}(t)=-\cos m_{2} t, x_{3}(t)=-\sin m_{2} t,
$$

where $m_{1}=\sqrt{\frac{k-\sqrt{k^{2}-4 l}}{2}}, m_{2}=\sqrt{\frac{k+\sqrt{k^{2}-4 l}}{2}}$ Clearly, $m_{1} \in\left(0, \frac{\pi}{2}\right), m_{2} \in\left(0, \frac{\pi}{2}\right)$, $m_{1}<m_{2}$ and then

$$
\cos m_{1} t>0, \cos m_{2} t>0, \forall t \in[0,1] .
$$

It is easy to check that $x_{0}(t), x_{1}(t), x_{2}(t)$, and $x_{3}(t)$ form a basis of solutions of $L[x]=$ 0 .

By simple computation, we have

$$
w_{1}=\cos m_{1} t, w_{2}=m_{1}, w_{3}=m_{1}\left(m_{2}^{2}-m_{1}^{2}\right) \cos m_{2} t, w_{4}=m_{1} m_{2}\left(m_{2}^{2}-m_{1}^{2}\right)^{2}(2.4
$$

Clearly, $w_{i}>0(i=1,2,3,4)$ on $[0,1]$.

By Lemma $2.2, L[x]=0$ is disconjugate on $[0,1]$, and $L[x]$ has a factorization

$$
\begin{aligned}
& x^{\prime \prime \prime \prime}+k x^{\prime \prime}+l x \\
& =\frac{m_{2}\left(m_{2}^{2}-m_{1}^{2}\right)}{\cos m_{2} t}\left(\frac{\cos ^{2} m_{2} t}{m_{2}}\left(\frac{m_{1}}{\left(m_{2}^{2}-m_{1}^{2}\right) \cos m_{1} t \cos m_{2} t}\left(\frac{\cos ^{2} m_{1} t}{m_{1}}\left(\frac{1}{\cos m_{1} t} x\right)^{\prime}\right)^{\prime}\right)^{\prime}\right)^{\prime}
\end{aligned}
$$

and accordingly

$$
L_{0} x=\rho_{0} x=\frac{1}{\cos m_{1} t} x, L_{1} x=\rho_{1}\left(L_{0} x\right)=\frac{1}{m_{1}}\left(\cos m_{1} t \cdot x^{\prime}+m_{1} \sin m_{1} t \cdot x\right) .
$$

Using (2.47), we conclude that $x(0)=x(1)=x^{\prime}(0)=x^{\prime}(1)=0$ is equivalent to (2.8).

This completes the proof of Theorem 2.4 .

Remark 2.5. If condition $(A 1)$ does not hold, the results of Theorem 2.4 cannot be obtained. For example, in the case of $L[x]=0$ with $k=-\pi^{2}, l=\pi^{4}, t=\frac{5}{6} \in[0,1]$, we have $l>\frac{1}{4}\left(k-\frac{\pi^{2}}{4}\right)^{2}, k \in(-\infty, 0)$. Applying the similar method to prove case 1 in 
Theorem 2.4, we conclude that

$$
x_{0}(t)=e^{-\frac{\sqrt{3 \pi}}{2} t} \cos \frac{\pi}{2} t, x_{1}(t)=e^{-\frac{\sqrt{3 \pi}}{2} t} \sin \frac{\pi}{2} t, x_{2}(t)=e^{-\frac{\sqrt{3 \pi}}{2} t} \cos \frac{\pi}{2} t, x_{3}(t)=e^{-\frac{\sqrt{3 \pi}}{2} t} \sin \frac{\pi}{2} t
$$

form a basis of solutions of $L[x]=0$. By simple computation, we have

$$
w_{1}=\frac{\cos \frac{\pi}{2} t}{e \frac{\sqrt{3 \pi}}{2} t}, w_{2}=\frac{\pi}{2} e^{-\sqrt{3} \pi t}, w_{3}=\frac{\sqrt{3}}{2} \pi^{3}\left(\sqrt{3} \cos \frac{\pi}{2} t-\sin \frac{\pi}{2} t\right) e^{-\frac{\sqrt{3} \pi}{2} t}, w_{4}=3 \pi^{6} .
$$

From $\frac{\pi}{2} t=\frac{5}{12} \pi$, we easily get that $\tan \frac{\pi}{2} t=2+\sqrt{3}>\sqrt{3}$. Furthermore, $w_{3}<0$. Thus, Theorem 2.4 does not hold in this case.

Remark 2.6. In the following, consider $L[x]=0$, for $k, l$ are given constants, by the similar method in Remark 2.5, we may gain the location of $(k, l)$ in the $(k, l)$-plane and the results of $w_{3}$ or $w_{1}$ corresponding $k, l: k=\frac{\pi^{2}}{16}, l=\frac{4 \pi^{2}}{16^{2}}, t=\frac{2}{\sqrt{5}}, l>\frac{1}{4}\left(k-\frac{\pi^{2}}{4}\right)^{2}$ and $w_{3}<0 ; k=\frac{\pi^{2}}{16}, l=\frac{9 \pi^{4}}{4 \times 16^{2}}, t=1, l=\frac{1}{4}\left(k-\frac{\pi^{2}}{4}\right)^{2}$ and $w_{3}<0 ; k=\frac{\pi^{2}}{16}, l=\frac{\pi^{4}}{16^{2}}, t=\frac{4}{3 \sqrt{3}}, \frac{1}{4} k^{2}<l<\frac{1}{4}\left(k-\frac{\pi^{2}}{4}\right)^{2}$ and $w_{3}=0 ; k=\pi^{2}, l=\frac{\pi^{4}}{4}, t=\frac{2 \sqrt{2}}{3}, l=\frac{1}{4} k^{2}$ and $w_{1}<0 ; k=\pi^{2}, l=\frac{\pi^{4}}{8}, t=\sqrt{2-\sqrt{2}}, 0<l<\frac{1}{4} k^{2}$ and $w_{3}<0 ; k=4 \pi^{2}, l=0, t=\frac{3}{4}$ and $w_{3}<0 ; k=\frac{\pi^{2}}{2}, l=-\frac{\pi^{4}}{2}, t=1, l=\pi^{2}\left(k-\pi^{2}\right)$ and $w_{3}=0 ; k=-\pi^{2}, l=-8 \pi^{4}, t=\frac{\sqrt{2}}{2}, l<\pi^{2}\left(k-\pi^{2}\right)$ and $\omega_{3}<0$. Furthermore, it follows that the conclusion of Theorem 2.4 cannot be yielded in the cases.

Theorem 2.7. Let (A1) hold and h satisfy (H2). Then

(i) The problem (1.8) has an infinite number of positive eigenvalue

$$
\lambda_{1}(h)<\lambda_{2}(h)<\cdots<\lambda_{k}(h)<\lambda_{k+1}(h)<\cdots .
$$

(ii) $\lambda_{k}(h) \rightarrow \infty$ as $k \rightarrow \infty$.

(iii) To each eigenvalue $\lambda_{k}(h)$ there corresponds an essential unique eigenfunction $\psi_{k}$ which has exactly $k-1$ simple zeros in $(0,1)$ and is positive near 0 .

(iv) Given an arbitrary subinterval of [0,1], then an eigenfunction which belongs to a sufficiently large eigenvalue change its sign in that subinterval.

(v) For each $k \in \mathbb{N}$, the geometric multiplicity of $\lambda_{k}(h)$ is 1 .

Proof of Theorem 2.7. (i)-(iv) are immediate consequences of Elias [[7], Theorem 1-5] and Theorem 2.4, we only prove (v).

Let

$$
\hat{L} x:=x^{\prime \prime \prime \prime}+k x^{\prime \prime}+l x, x \in D(\hat{L})
$$

with

$$
D(\hat{L})=\left\{x \in C^{4}[0,1] \mid x(0)=x(1)=x^{\prime}(0)=x^{\prime}(1)=0\right\} .
$$

To show $(v)$, it is enough to prove

$$
\operatorname{ker}\left(\hat{L}-\lambda_{k}(h) h(\cdot)\right)^{2}=\operatorname{ker}\left(\hat{L}-\lambda_{k}(h) h(\cdot)\right) .
$$


Clearly

$$
\operatorname{ker}\left(\hat{L}-\lambda_{k}(h) h(\cdot)\right)^{2} \supseteq \operatorname{ker}\left(\hat{L}-\lambda_{k}(h) h(\cdot)\right) .
$$

Suppose on the contrary that the geometric multiplicity of $\lambda_{k}(h)$ is greater than 1 . Then there exists $x \in \operatorname{ker}\left(\hat{L}-\lambda_{k}(h) h(\cdot)\right)^{2} \backslash \operatorname{ker}\left(\hat{L}-\lambda_{k}(h) h(\cdot)\right)$ and subsequently

$$
\hat{L} x-\lambda_{k}(h) h(t) x=\gamma \psi_{k}
$$

for some $\gamma \neq 0$. Multiplying both sides of (2.50) by $\psi_{k}(t)$ and integrating from 0 to 1 , we deduce that

$$
0=\gamma \int_{0}^{1}\left[\psi_{k}(t)\right]^{2} d t
$$

which is a contradiction !

Theorem 2.8 (Maximum principle). Let $(A 1)$ hold. Let $e \in C[0,1]$ with $e \geq 0$ on $[0,1]$ and $e \neq 0$ on any compact subinterval in $[0,1]$. If $x \in C^{4}[0,1]$ satisfies

$$
\begin{aligned}
& x^{\prime \prime \prime \prime}+k x^{\prime \prime}+l x=e(t), 0<t<1, \\
& x(0)=x(1)=x^{\prime}(0)=x^{\prime}(1)=0,
\end{aligned}
$$

Then $x>0$ on $(0,1)$.

Proof. When $(A 1)$ holds, the homogeneous problem

$$
\begin{aligned}
& x^{\prime \prime \prime \prime}+k x^{\prime \prime}+l x=0,0<t<1, \\
& x(0)=x(1)=x^{\prime}(0)=x^{\prime}(1)=0
\end{aligned}
$$

has only trivial solution. So the boundary value problem (2.52) has a unique solution which may be represented in the form

$$
x(t)=\int_{0}^{1} G(t, s) e(s) d s,
$$

where $G(t, s)$ is Green's function. By Theorem 2.4 and Lemma 2.3 (take $n=4, k=2$ ), we have

$$
(-1)^{4-2} G(t, s)>0, \forall(t, s) \in(0,1) \times(0,1),
$$

that is, $G(t, s)>0$, for all $(t, s)\lfloor(0,1) \times(0,1)$.

Using (2.54), when $e \geq 0$ on $[0,1]$ and $e \neq 0$ on any compact subinterval in $[0,1]$, then $x>0$ on $(0,1)$.

\section{Main results}

Theorem 3.1. Let (A1), (A2), (A3) and (A4) hold. Assume that either (i) or (ii) holds for some $k \in \mathbb{N}$ and $j \in\{0\} \cup \mathbb{N}$ :

$$
\begin{aligned}
& (i) \lambda_{k}(b)<\cdots<\lambda_{k+j}(b)<1<\lambda_{k}(a)<\cdots<\lambda_{k+j}(a) ; \\
& (i i) \lambda_{k}(a)<\cdots<\lambda_{k+j}(a)<1<\lambda_{k}(b)<\cdots<\lambda_{k+j}(b) .
\end{aligned}
$$

Then the problem $(1.2)$ has $2(j+1)$ solutions $x_{k+i^{\prime}}^{+} x_{k+i^{\prime}}^{-} i=0, \ldots, j, x_{k+i}^{+}$has exactly $k+i-$ 1 zeros in $(0,1)$ and is positive near $t=0$, and $x_{k+i}^{-}$has exactly $k+i-1$ zeros in $(0,1)$ and is negative near $t=0$. 
Theorem 3.2 Let (A1), (A2), (A3) and (A5) hold. Assume that for some $k \in \mathbb{N}$,

$$
\lambda_{k}(a)<1 .
$$

Then there are at least $2 k-1$ nontrivial solutions of the problem (1.2). In fact, there exist solutions $\omega_{1}, \ldots, \omega_{k}$ such that for $1 \leq j \leq k, \omega_{j}$ has exactly $j-1$ simple zeros on the open interval $(0,1)$ and $\omega_{j}^{\prime \prime}(0)<0$ and there exist solutions $z_{2}, \ldots, z_{k}$ such that for $2 \leq j \leq$ $k, z_{j}$ has exactly $j-1$ simple zeros on the open interval $(0,1)$ and $z_{j}^{\prime \prime}(0)>0$.

Let $Y=C[0,1]$ with the norm

$$
\|x\|_{\infty}=\max _{t \in[0,1]}|x| .
$$

Let $E=\left\{x \in C^{2}[0,1] \mid x(0)=x(1)=x^{\prime}(0)=x^{\prime}(1)=0\right\}$ with the norm

$$
\|x\|_{E}=\max \left\{\|x\|_{\infty},\left\|x^{\prime}\right\|_{\infty^{\prime}}\left\|x^{\prime \prime}\right\|_{\infty}\right\} .
$$

Then $\hat{L}^{-1}: Y \rightarrow E$ is completely continuous. Here $\hat{L}$ is given as in (2.48).

Let $\zeta(\cdot),, \xi_{1}(\cdot),, \xi_{2}(\cdot, \cdot) \in C([0,1] \times \mathbb{R}, \mathbb{R})$ be such that

$$
\begin{gathered}
f(t, x)=a(t) x+\zeta(t, x), f(t, x)=b(t) x+\xi_{1}(t, x), \\
f(t, x)=c(t) x^{+}+\xi_{2}(t, x), \forall(t, x) \in[0,1] \times \mathbb{R} .
\end{gathered}
$$

Here $x^{+}=\max \{x, 0\}$.

Clearly,

$$
\lim _{|x| \rightarrow 0} \frac{\zeta(t, x)}{x}=0, \lim _{|x| \rightarrow \infty} \frac{\xi_{1}(t, x)}{x}=0, \lim _{|x| \rightarrow \infty} \frac{\xi_{2}(t, x)}{x}=0
$$

uniformly for $t \in[0,1]$.

Let

$$
\begin{aligned}
& \bar{\xi}_{1}(x)=\max \left\{\left|\xi_{1}(t, s)\right|: 0 \leq|s| \leq x, t \in[0,1]\right\}, \\
& \bar{\xi}_{2}(x)=\max \left\{\left|\xi_{2}(t, s)\right|: 0 \leq|s| \leq x, t \in[0,1]\right\},
\end{aligned}
$$

then $\bar{\xi}_{1}$ and $\bar{\xi}_{2}$ are nondecreasing and

$$
\lim _{x \rightarrow \infty} \frac{\bar{\xi}_{1}(x)}{x}=0, \lim _{x \rightarrow \infty} \frac{\bar{\xi}_{2}(x)}{x}=0 .
$$

Let us consider

$$
\hat{L} x=\lambda a(t) x+\lambda \zeta(t, x)
$$

as a bifurcation problem from the trivial solution $x \equiv 0$.

Equation (3.9) can be converted to the equivalent equation

$$
x(t)=\lambda \hat{L}^{-1}[a(\cdot) x(\cdot)](t)+\lambda \hat{L}^{-1}[\zeta(\cdot, x(\cdot))](t) .
$$

Clearly, the compactness of $\hat{L}^{-1}$ together with (3.6) imply that

$$
\left\|\hat{L}^{-1}[\zeta(\cdot, x(\cdot))]\right\|_{E}=o(\|x\|)_{E}, a s\|x\|_{E} \rightarrow 0 .
$$

Let $S_{k}^{+}$denotes the set of functions in $E$ which have exactly $k$ - 1 interior nodal (i.e., non-degenerate) zeros in $(0,1)$ and are positive near $t=0$, set $S_{k}^{-}=-S_{k}^{+}$, and 
$\Phi_{k}^{ \pm}=\mathbb{R} \times S_{k}^{ \pm}$. They are disjoint and open sets in $E$. Finally, let $\Phi_{k}^{ \pm}=\mathbb{R} \times S_{k}^{ \pm}$and $\Phi_{k}=$ $\mathbb{R} \times S_{k}$.

The results of Rabinowitz [15] for (3.9) can be stated as follows: For each integer $k \geq$ 1 and each $v=\{+,-\}$, there exists a continuum $C_{k}^{v} \subseteq \Phi_{k}^{v}$ of solution of (3.9), joining ( $\lambda_{k}$ $(a), 0)$ to infinity in $\Phi_{k}^{v}$. Moreover, $C_{k}^{v} \backslash\left(\lambda_{k}(a), 0\right) \subset \Phi_{k^{*}}^{v}$

Notice that we have used the fact that if $x$ is a nontrivial solution of (3.9), then all zeros of $x$ on $(0,1)$ are simple under $(A 1),(A 2),(A 3)$, and $(A 4)$.

In fact, (3.9) can be rewritten to

$$
\hat{L} x=\lambda \hat{a}(t) x,
$$

where

$$
\hat{a}(t)=\left\{\begin{array}{l}
\frac{f(t, x(t))}{x(t)}, x(t) \neq 0, \\
a(t), x(t)=0 .
\end{array}\right.
$$

Clearly $\hat{a}(t)$ satisfies $(H 2)$. So Theorem 2.7 (iii) yields that all zeros of $x$ on $(0,1)$ are simple.

Proof of Theorem 3.1. We first prove the theorem when $j=0$.

It is clear that any solution of (3.9) of the form $(1, x)$ yields solutions $x$ of (1.2). We will show that $C_{k}^{v}$ crosses the hyperplane $\{1\} \times E$ in $\mathbb{R} \times E$. To do this, it is enough to show that $C_{k}^{v}$ joins $\left(\lambda_{k}(a), 0\right)$ to $\left(\lambda_{k}(b), \infty\right)$. Let $\left(\mu_{n}, x_{n}\right) \in C_{k}^{v}$ satisfy

$$
\mu_{n}+\left\|x_{n}\right\|_{E} \rightarrow \infty
$$

We note that $\mu_{n}>0$ for all $n \in \mathbb{N}$ since $(0,0)$ is the only solution of (3.9) for $\lambda=0$ and $C_{k}^{v} \cap(\{0\} \times E=\varnothing$.

Case 1. $\lambda_{k}(b)<1<\lambda_{k}(a)$.

In this case, we show that

$$
\left(\lambda_{k}(b), \lambda_{k}(a)\right) \subseteq\left\{\lambda \in \mathbb{R}: \exists(\lambda, x) \in C_{k}^{\nu}\right\} .
$$

We divide the proof into two steps.

Step 1 . We show that if there exists a constant number $M>0$ such that

$$
\mu_{n} \in(0, M]
$$

then $C_{k}^{v}$ joins $\left(\lambda_{k}(a), 0\right)$ to $\left(\lambda_{k}(b), \infty\right)$.

In this case $\left\|x_{n}\right\|_{E} \rightarrow \infty$. We divide the equation

$$
\hat{L} x_{n}=\mu_{n} b(t) x_{n}+\mu_{n} \xi_{1}\left(t, x_{n}\right), t \in(0,1)
$$

by $\left\|x_{n}\right\|_{E}$ and set $y_{n}=\frac{x_{n}}{\left\|x_{n}\right\|_{E}}$. Since $y_{n}$ is bounded in $C^{2}[0,1]$, choosing a subsequence and relabeling if necessary, we have that $y_{n} \rightarrow y$ for some $y \in E$ with $\|y\|_{E}=1$. Moreover, from (3.8) and the fact that $\bar{\xi}_{1}$ is nondecreasing, we have that

$$
\lim _{n \rightarrow \infty} \frac{\left|\xi_{1}\left(t, x_{n}(t)\right)\right|}{\left\|x_{n}\right\|_{E}}=0, \forall t \in[0,1],
$$


since

$$
\frac{\left|\xi_{1}\left(t, x_{n}(t)\right)\right|}{\left\|x_{n}\right\|_{E}} \leq \frac{\bar{\xi}_{1}\left(\left|x_{n}(t)\right|\right)}{\left\|x_{n}\right\|_{E}} \leq \frac{\bar{\xi}_{1}\left(\left\|x_{n}(t)\right\|_{\infty}\right)}{\left\|x_{n}\right\|_{E}} \leq \frac{\bar{\xi}_{1}\left(\left\|x_{n}(t)\right\|_{E}\right)}{\left\|x_{n}\right\|_{E}}, \forall t \in[0,1] .
$$

Thus

$$
\gamma(t)=\hat{L}^{-1}[\mu b(\cdot) \gamma(\cdot)](t),
$$

where $\mu:=\lim _{n \rightarrow \infty} \mu_{n}$, again choosing a subsequence and relabeling if necessary. Thus

$$
\hat{L} y=\mu b(t) y .
$$

We claim that

$$
y \in S_{k}^{v}
$$

Suppose, to the contrary, that $y \notin S_{k}^{v}$. Since $y \neq 0$ is a solution of (3.18), all zeros of $y$ in $[0,1]$ are simple. It follows that $y \in S_{h}^{l} \neq S_{k}^{v}$ for some $h \in \mathbb{R}$ and $l \in\{+,-\}$. By the openness of $S_{h}^{l}$ we know that there exists a neighborhood $U\left(y, \rho_{0}\right)$ such that

$$
U\left(y, \rho_{0}\right) \subset S_{h^{\prime}}^{l}
$$

which, together with the fact $y_{n} \rightarrow y$, implies that exists $n_{0} \in \mathbb{N}$ such that

$$
y_{n} \in S_{h^{\prime}}^{l} n \geq n_{0} .
$$

However, this contradicts the fact that $y_{n} \in S_{k}^{v}$ Therefore, $y \in S_{k}^{v}$

Now, by Theorem 2.7, we obtain $\mu=\lambda_{k}(b)$.

Thus $C_{k}^{v}$ joins $\left(\lambda_{k}(a), 0\right)$ to $\left(\lambda_{k}(b), \infty\right)$.

Step 2. We show that there exists a constant number $M>0$ such that $\mu_{n} \in(0, M]$, for all $n$.

Suppose there is no such $M$. Choosing a subsequence and relabeling if necessary, it follows that

$$
\lim _{n \rightarrow \infty} \mu_{n}=\infty .
$$

Let

$$
0=\tau(0, n)<\tau(1, n)<\cdots<\tau(k, n)=1
$$

denotes the zeros of $x_{n}$. Then there exists a subsequence $\left\{\tau\left(1, n_{m}\right)\right\} \subseteq\{\tau(1, n)\}$ such that

$$
\lim _{m \rightarrow \infty} \tau\left(1, n_{m}\right):=\tau(1, \infty) .
$$

Clearly

$$
\lim _{m \rightarrow \infty} \tau\left(0, n_{m}\right):=\tau(0, \infty)=0 .
$$

We claim that

$$
\tau(1, \infty)-\tau(0, \infty)=0 .
$$


Suppose, to the contrary, that

$$
\tau(0, \infty)<\tau(1, \infty) .
$$

Define a function $p:[0,1] \times[0, \infty) \rightarrow \mathbb{R}$ by

$$
p(t, x)=\left\{\begin{array}{l}
\frac{f(t, x)}{x}, x \neq 0, t \in[0,1], \\
a(t), x=0, t \in[0,1] .
\end{array}\right.
$$

Then, by (A2), (A3), and (A4), there exist two positive numbers $\rho_{1}$ and $\rho_{2}$, such that

$$
\rho_{1} \leq \frac{f(t, x)}{x} \leq \rho_{2}, \text { for all } x \geq 0, t \in[0,1] .
$$

Using (3.22), (3.24), and the fact that $\lim _{m \rightarrow \infty} \mu_{n_{m}}=\infty$, we conclude that there exists a closed interval $I_{1} \subset(\tau(0, \infty), \tau(1, \infty))$ such that

$$
\lim _{m \rightarrow \infty} \mu_{n_{m}} p\left(t, x_{n_{m}}(t)\right)=\infty
$$

uniformly for $t \in I_{1}$.

However, since $x_{n_{m}}$ satisfies

$$
\hat{L} x_{n_{m}}(t)=\mu_{n_{m}} p\left(t, x_{n_{m}}(t)\right) x_{n_{m}}(t),
$$

the proof of Lemma 4 in [7] (see also the remarks in the final paragraph in [[7], p. 43]), shows that for all $n$ sufficiently large, $x_{n_{m}}$ must change sign on $I_{1}$. However, this contradicts the fact that for all $m$ sufficiently large we have $I_{1} \subset\left(\tau\left(0, n_{m}\right), \tau\left(1, n_{m}\right)\right)$ and

$$
v x_{n_{m}}(t)>0, t \in\left(\tau\left(0, n_{m}\right), \tau\left(1, n_{m}\right)\right) .
$$

Thus, (3.21) holds.

Next, we work with $\left(\tau\left(1, n_{m}\right), \tau\left(2, n_{m}\right)\right)$. It is easy to see that there is a subsequence $\tau\left(2, n_{m_{j}}\right) \subseteq \tau\left(2, n_{m}\right)$ such that

$$
\lim _{j \rightarrow \infty} \tau\left(2, n_{m_{j}}\right):=\tau(2, \infty) .
$$

Clearly

$$
\lim _{j \rightarrow \infty} \tau\left(1, n_{m_{j}}\right)=\tau(1, \infty) .
$$

We claim that

$$
\tau(2, \infty)-\tau(1, \infty)=0
$$

Suppose, to the contrary, that $\tau(1, \infty)<\tau(2, \infty)$. Then, from (3.23), (3.24), and the fact that $\lim _{j \rightarrow \infty} \mu_{n_{m_{j}}}=\infty$, there exists a closed interval $I_{2} \subset(\tau(1, \infty), \tau(2, \infty))$ such that

$$
\lim _{j \rightarrow \infty} \mu_{n_{m_{j}}} p\left(t, x_{n_{m_{j}}}(t)\right)=\infty
$$

uniformly for $t \in I_{2}$.

This implies the solution $x_{n_{m_{j}}}$ of the equation

$$
\hat{L} x_{n_{m_{j}}}(t)=\mu_{n_{m_{j}}} p\left(t, x_{n_{m_{j}}}(t)\right) x_{n_{m_{j}}}(t)
$$


must change sign on $I_{2}$. However, this contradicts the fact that for all $j$ sufficiently large we have $I_{2} \subset\left(\tau\left(1, n_{m_{j}}\right), \tau\left(2, n_{m_{j}}\right)\right)$ and

$$
v x_{n_{m_{j}}}(t)>0, t \in\left(\tau\left(1, n_{m_{j}}\right), \tau\left(2, n_{m_{j}}\right)\right) .
$$

Therefore, (3.26) holds.

By a similar argument to obtain (3.21) and (3.26), we can show that for each $l \in$ $\{2, \ldots, k-1\}$,

$$
\tau(l+1, \infty)-\tau(l, \infty)=0 .
$$

Taking a subsequence and relabeling it as $\left\{\left(\mu_{n}, x_{n}\right)\right\}$, if necessary, it follows that for each $l \in\{0, \ldots, k-1\}$,

$$
\lim _{n \rightarrow \infty}(\tau(l+1, n)-\tau(l, n))=0 .
$$

But this is impossible since

$$
1=\tau(k, n)-\tau(0, n)=\sum_{l=0}^{k-1}(\tau(l+1, n)-\tau(l, n))
$$

for all $n$. Therefore,

$$
\left|\mu_{n}\right| \leq M
$$

for some constant number $M>0$, independent of $n \in \mathbb{N}$.

Case 2. $\lambda_{k}(a)<1<\lambda_{k}(b)$.

In this case, if $\left(\mu_{n}, x_{n}\right) \in C_{k}^{v}$ is such that

$$
\lim _{n \rightarrow \infty}\left(\mu_{n}+\left\|x_{n}\right\|_{E}\right)=\infty
$$

and

$$
\lim _{n \rightarrow \infty} \mu_{n}=\infty,
$$

then

$$
\left(\lambda_{k}(a) m, \lambda_{k}(b)\right) \subseteq\left\{\lambda \in(0, \infty) \mid(\lambda, x) \in C_{k}^{\nu}\right\}
$$

and, moreover, $(\{1\} \times E) \cap \mathcal{C}_{k}^{v} \neq \varnothing$.

Assume that there exists $M>0$ such that for all $n \in \mathbb{N}$,

$$
\mu_{n} \in(0, M] .
$$

Applying a similar argument to that used in step 1 of Case 1 , after taking a subsequence and relabeling if necessary, it follows that

$$
\left(\mu_{n}, x_{n}\right) \rightarrow\left(\lambda_{k}(b), \infty\right), n \rightarrow \infty .
$$

Again $C_{k}^{v}$ joins $\left(\lambda_{k}(a), 0\right)$ to $\left(\lambda_{k}(b), \infty\right)$ and the result follows.

Finally, let $j \in \mathbb{N}$. By repeating the arguments used in the proof of the case $j=0$, we see that for each $v \in\{+,-\}$ and each $i \in\{k, k+1, \ldots, k+j\}$,

$$
C_{i}^{v} \cap(\{1\} \times E) \neq \varnothing .
$$

The result follows. 
Proof of Theorem 3.2.

We only need to show that

$$
\begin{aligned}
& C_{j}^{-} \cap(\{1\} \times E) \neq \emptyset, j=1,2, \ldots, k, \\
& C_{j}^{+} \cap(\{1\} \times E) \neq \emptyset, j=2, \ldots, k .
\end{aligned}
$$

Suppose on the contrary that

$$
C_{i}^{l} \cap(\{1\} \times E)=\emptyset \text {, for some }(i, l) \in \Gamma,
$$

where

$$
\Gamma:=\{(j, v) \mid j \in\{2, \ldots, k\} \text { as } v=+, j \in\{1,2, \ldots, k\} \text { as } v=-\} \text {. }
$$

Since $C_{i}^{l}$ joins $\left(\lambda_{i},(a), 0\right)$ to infinity in $\Phi_{i}^{l}$ and $(\lambda, x)=(0,0)$ is the unique solution of $(3.9)_{\lambda=0}$ in $E$, there exists a sequence $\left\{\left(\mu_{n}, x_{n}\right)\right\} \subset C_{i}^{l}$ such that $\mu_{n} \in(0,1)$ and $\left\|x_{n}\right\|_{E}$ $\rightarrow \infty$ as $n \rightarrow \infty$. We may assume that $\mu_{n} \rightarrow \mu \in[0,1]$ as $n \rightarrow \infty$. Let $y_{n}=x_{n} /\left\|_{x_{n}}\right\|_{E} n \geq$ 1. From the fact

$$
\hat{L} x_{n}(t)=\mu_{n} c(t)\left(x_{n}\right)^{+}(t)+\mu_{n} \xi_{2}\left(t, x_{n}(t)\right) .
$$

We have that

$$
y_{n}(t)=\mu_{n} \hat{L}^{-1}\left[c(\cdot)\left(y_{n}\right)^{+}\right](t)+\mu_{n} \hat{L}^{-1}\left[\frac{\xi_{2}\left(\cdot, x_{n}\right)}{\left\|x_{n}\right\|_{E}}\right](t) .
$$

Furthermore, since $\left.\hat{L}^{-1}\right|_{E}: E \rightarrow E$ is completely continuous, we may assume that there exists $y \in E$ with $\|y\|_{E}=1$ such that $\left\|y_{n}-y\right\|_{E} \rightarrow 0$ as $n \rightarrow \infty$. Since

$$
\frac{\left|\xi_{2}\left(t, x_{n}\right)\right|}{\left\|x_{n}\right\|_{E}} \leq \frac{\bar{\xi}_{2}\left(\left\|x_{n}\right\|_{\infty}\right)}{\left\|x_{n}\right\|_{E}} \leq \frac{\bar{\xi}_{2}\left(\left\|x_{n}\right\|_{E}\right)}{\left\|x_{n}\right\|_{E}}
$$

uniformly for $t \in[0,1]$, we have from (3.39) and (3.8) that

$$
y=\mu \hat{L}^{-1}\left[c(t) y^{+}\right]
$$

that is,

$$
\begin{gathered}
y^{\prime \prime \prime \prime}+k y^{\prime \prime}+l y=\mu c(t) y^{+}, 0<t<1, \\
y(0)=y(1)=y^{\prime}(0)=y^{\prime}(1)=0 .
\end{gathered}
$$

By $(A 1),(A 5)$, and (3.42) and the fact that $\|y\|_{E}=1$, we conclude that $\mu c(t) y^{+} \neq 0$ on any compact subinterval in $[0,1]$, and consequently

$$
\left.\mu>0, y^{+}\right) \not \equiv 0 \text {, on any compact subinterval in }[0,1] \text {. }
$$

By Theorem 2.8, we know that $y(t)>0$ in $(0,1)$. This means $\mu$ is the first eigenvalue of $\hat{L} x=\lambda c(t) x$ and $y$ is the corresponding eigenfunction. Hence $y \in S_{1}^{+}$and therefore, since $S_{1}^{+}$is open and $\left\|y_{n}-y\right\|_{E} \rightarrow 0$, we have that $y_{n} \in S_{1}^{+}$for $n$ large. But this contradicts the assumption that $\left(\mu_{n}, y_{n}\right) \in C_{i}^{l}$ and $(i, l) \in \Gamma$, so (3.36) is wrong, which completes the proof. 


\section{Acknowledgements}

Thanks are given to Professor R.Y. Ma for his valuable suggestion. The author is also grateful to the anonymous referee for his/her valuable suggestions. This study was supported by: the NSFC (No. 11031003); the Scientific Research Foundation of the Education department of Gansu Province (No. 1114-04).

\section{Author details}

${ }^{1}$ School of Mathematics and Statistics, Lanzhou University, Lanzhou 730000, People's Republic of China ${ }^{2}$ Department of Basic Courses, Lanzhou Polytechnic College, Lanzhou 730050, People's Republic of China

\section{Competing interests}

The author declares that they have no competing interests.

Received: 15 August 2011 Accepted: 20 March 2012 Published: 20 March 2012

\section{References}

1. Agarwal, RP, Chow, YM: Iterative methods for a fourth-order boundary value problem. J Comput Appl Math. 10(2):203-217 (1984). doi:10.1016/0377-0427(84)90058-X

2. $\mathrm{Ma}, \mathrm{R}, \mathrm{Wu}, \mathrm{HP}$ : Positive solutions of a fourth-order two-point boundary value problem. Acta Math Sci A. 22(2):244-249 (2002)

3. Yao, Q: Positive solutions for eigenvalue problems of fourth-order elastic beam equations. Appl Math Lett. 17(2):237-243 (2004). doi:10.1016/50893-9659(04)90037-7

4. Yao, Q: Solvability of an elastic beam equation with Caratheodory function. Math Appl. 17(3):389-392 (2004)

5. Korman, P: Uniqueness and exact multiplicity of solutions for a class of fourth-order semilinear problems. Proc Royal Soc Edinburgh A. 134(1):179-190 (2004). doi:10.1017/\$0308210500003140

6. $\mathrm{Xu}, J, \mathrm{Han}, \mathrm{X}$ : Nodal solutions for a fourth-order two-point boundary value problem. Boundary Value Problem 2010, 11 (2010). Article ID 570932

7. Elias, U: Eigenvalue problems for the equations $L y+\lambda p(x) y=0$. J Diff Equ. 29(1):28-57 (1978). doi:10.1016/0022-0396(78) 90039-6

8. Elias, U: Oscillation Theory of Two-Term Differential Equations, vol. 396 of Mathematics and Its Applications. Kluwer Academic Publishers, Dordrecht, The Netherlands (1997)

9. Ma, R: Nodal Solutions for a fourth-order two-point boundary value problem. J Math Anal Appl. 314(1):254-265 (2006). doi:10.1016/j.jmaa.2005.03.078

10. Ma, R: Nodal Solutions of boundary value problem of fourth-order ordinary differential equations. J Math Anal Appl. 319(2):424-434 (2006). doi:10.1016/j.jmaa.2005.06.045

11. $\mathrm{Ma}, \mathrm{R}, \mathrm{Xu}, \mathrm{J}$ : Bifurcation from interval and positive solutions of a fourth-order boundary value problem. Nonlinear Anal Theory Methods Appl. 72(1):113-122 (2010). doi:10.1016/j.na.2009.06.061

12. $\mathrm{Ma}, \mathrm{R}$, Wang, $\mathrm{H}$ : On the existence of positive solutions of a fourth-order ordinary differential equations. Appl Anal. 59(14):225-231 (1995). doi:10.1080/00036819508840401

13. Ma, R: Existence of positive solutions of a fourth-order boundary value problem. Appl Math Comput. 168(2):1219-1231 (2005). doi:10.1016/j.amc.2004.10.014

14. Ma, R, Thompson, B: Nodal solutions for a nonlinear fourth-order eigenvalue problem. Acta Math Sin (Engl Ser). 24(1):27-34 (2008). doi:10.1007/s10114-007-1009-6

15. Rabinowitz, PH: Some global results for nonlinear eigenvalue problems. J Funct Anal. 7(32):487-513 (1971)

doi:10.1186/1687-2770-2012-31

Cite this article as: Shen: Existence of nodal solutions of a nonlinear fourth-order two-point boundary value problem. Boundary Value Problems 2012 2012:31.

\section{Submit your manuscript to a SpringerOpen ${ }^{\circ}$ journal and benefit from:}

- Convenient online submission

Rigorous peer review

- Immediate publication on acceptance

- Open access: articles freely available online

- High visibility within the field

- Retaining the copyright to your article

Submit your next manuscript at $\boldsymbol{s p r i n g e r o p e n . c o m ~}$ 